Faculty of Economics and Social Sciences

Department of Economics

\title{
Adversarial versus Inquisitorial Testimony
}

Winand Emons

Claude Fluet

09-04

September 2009

\section{DISCUSSION PAPERS}




\title{
Adversarial versus Inquisitorial Testimony*
}

\author{
Winand Emons ${ }^{\dagger} \quad$ Claude Fluet ${ }^{\ddagger}$ \\ Universität Bern Université du Québec \\ CEPR à Montréal, CIRPEE
}

Version: September 2009

\begin{abstract}
An arbiter can decide a case on the basis of his priors, or the two parties to the conflict may present further evidence. The parties may misrepresent evidence in their favor at a cost. At equilibrium the two parties never testify together. When the evidence is much in favor of one party, this party testifies. When the evidence is close to the prior mean, no party testifies. We compare this outcome under a purely adversarial procedure with the outcome under a purely inquisitorial procedure (Emons and Fluet 2009). We provide sufficient conditions on when one procedure is better than the other one.
\end{abstract}

Keywords: evidence production, procedure, costly state falsification, adversarial, inquisitorial.

JEL: D82, K41, K42

\section{Introduction}

How much testimony will an arbiter hear in adversarial proceedings when the parties to the conflict may spend resources to misrepresent evidence in

\footnotetext{
${ }^{*}$ We thank Simon Lörtscher for helpful comments. The usual disclaimer applies.

†Departement Volkswirtschaftslehre, Universität Bern, Schanzeneckstrasse 1, Postfach 8573, CH-3001 Bern, Switzerland, Phone: +41-31-6313922, Fax: +41-31-6313383, Email: winand.emons@vwi.unibe.ch.

¥Université du Québec à Montréal and CIRPÉE, C.P. 8888, Suc. Centre-Ville, Montréal H3C 3P8, Canada, Phone: +1-514-9878386, Fax: +1-514-9878494, Email: fluet.claudedenys@uqam.ca.
} 
their favor? Will both parties come forward with boosted claims offsetting each other, or will only the party for whom the evidence is favorable testify? Are there circumstances where no party testifies? What are the efficiency properties of the outcome? Is the adversarial procedure where the parties to the conflict decide whether or not they testify better than the inquisitorial procedure where the arbiter decides how much testimony he wants to hear? In this paper we address these questions.

An arbiter has to decide on an issue which we take to be a real number, for example, the damages that one party owes to the other. The defendant wants the damages awarded to be small whereas the plaintiff wants them to be large. Both parties know the actual amount owed to the plaintiff and both would like to influence the arbiter's decision. Presenting evidence, however, involves a fixed cost. Moreover, the parties can boost the evidence in either direction, but distorting the evidence involves additional costs: the greater the distortion, the higher the cost. For instance, expert witnesses charge more the more they distort the truth.

In a purely adversarial procedure the parties decide whether or not to present testimony. The arbiter is passive at the discovery stage and only intervenes at the adjudication stage. Once the parties have finished, he decides the case on the basis of his priors about the amount at issue and of what can be inferred from the parties' actions. The arbiter seeks to minimize adjudication error, implying that his sequentially rational decision is to adjudicate the posterior mean. When he hears no testimony, given the symmetry of the parties' actions, the posterior mean equals the prior, which the arbiter therefore adjudicates. When he hears testimony, he updates his beliefs upwards or downwards and adjudicates accordingly.

We first show that in equilibrium the parties never testify together: either no or one party testifies. No party testifies when the true value is close to the prior mean and thus influencing the arbiter has negligible private value: it does not pay for the parties to incur the fixed cost of testifying. When, however, the evidence is sufficiently in favor of one party, this party comes 
forward and testifies. If the plaintiff testifies, he overstates the true value; if the defendant testifies, he understates the true value. Boasting increases the more the true amount differs from the prior mean, yet at a decreasing rate. Accordingly, for sufficiently large deviations from the prior mean, the equilibrium is revealing, but it involves falsification on the part of the party who testifies. The arbiter rationally corrects for the exaggerated amount and adjudicates the true value. Stated differently, because the marginal cost of slightly distorting the truth is negligible but the marginal return is not, the arbiter expects some falsification, leading parties to do so systematically. At equilibrium the arbiter expects both, error and falsification costs.

We measure welfare by summing the social loss from inaccurate adjudication and the parties' submission costs. From a welfare point of view the adversarial procedure has the following virtues: when the true value is close to the prior mean, the parties remain silent and do not spend resources on falsification, yet at the expense of incorrect decisions. Only when the true value differs sufficiently from the prior mean do parties testify, thus enabling correct decisions, yet at the expense of falsification.

In a second step we compare the equilibrium for the adversarial procedure with our results for the purely inquisitorial procedure as derived in Emons and Fluet (2009). Under the purely inquisitorial procedure the arbiter plays first by deciding how much testimony he wants to hear. We assume that the inquisitorial arbiter seeks to maximize welfare, taking into account submission costs and adjudication error. Specifically, he first announces whether he wants to hear no, one, or both parties. The parties have to testify when called upon by the arbiter and cannot testify unless they are asked to do so. When the arbiter decides to hear no party, he rationally adjudicates the prior mean; there is no falsification but error costs are positive. When he decides to hear one party, depending on who testifies, the party over- resp. understates the true value. The arbiter rationally corrects for the exaggerated amount and adjudicates the true value. Accordingly, the equilibrium is revealing but it involves falsification. When both parties submit, both testimonies involve 
falsification: one party over-reports while the other under-reports. The arbiter corrects for this by taking an average of the exaggerated testimonies. Accordingly, under the inquisitorial procedure the arbiter incurs only error costs (no testimony) or only falsification costs (single or joint testimony).

The optimal number of parties to submit evidence depends on the weight given to inaccurate adjudication and the cost of obtaining evidence. If the value of accuracy is above some threshold, the arbiter hears both parties when the fixed cost of presenting evidence is small. For intermediate values of the fixed cost, the arbiter goes for one party, and for large values he hears no party at all.

Next we compare welfare under the two procedures. The inquisitorial procedure fares better than the adversarial one when the arbiter cares little or when he cares a lot about error costs. If error costs are of minor importance, under the inquisitorial procedure the arbiter goes for no testimony so that there are no falsification but only error costs. Under the adversarial procedure there is some range where parties testify so that there are falsification costs. When the arbiter cares a lot about error costs, he chooses single or joint testimony under the inquisitorial procedure so that he incurs no error costs. Under the adversarial procedure there is some range where the parties do not testify so that there are error costs. Accordingly, when the arbiter has strong views about error costs, the inquisitorial procedure does better because it gives the arbiter full control over what kind of cost he incurs.

Next we derive conditions for the adversarial procedure to be better than the inquisitorial one. If incorrect decisions do not matter too much and the fixed cost of testifying is sufficiently large, the adversarial procedure is better than the inquisitorial one. The reason for this is as follows: For the inquisitorial procedure no testimony is optimal under these conditions. For intermediate deviations from the prior mean, the parties do not testify under the adversarial procedure, hence we have the same outcome as under the inquisitorial procedure. Nevertheless, for large deviations the parties come forward and testify. For these large deviations, the falsification cost is lower 
than the cost of incorrect decisions.

If the fixed submission cost is above some threshold, single testimony is optimal under the inquisitorial procedure. When inaccurate decisions do not matter too much, the adversarial procedure again does better than the inquisitorial one. The reason is now that boasting is lower under adversarial than under inquisitorial testimony required from a single party. Finally, when the fixed cost of testifying is small, so is the range where parties do not testify under the adversarial procedure. The loss from inaccurate decisions is then lower than the submission costs under the inquisitorial procedure.

We thus have a simple framework allowing us to compare pure forms of the adversarial and inquisitorial procedures. Under either procedure, parties may dissipate resources in influence activities. Our approach is to treat boosted claims as costly signals. For the purpose of comparison we, therefore, select for each procedure the least-cost signalling equilibrium. In the adversarial procedure, the parties can submit evidence as they see fit. In the inquisitorial procedure, they can present evidence only if specifically required to do so. In either case, at the adjudication stage, the arbiter chooses the sequentially rational action given his beliefs. Under the adversarial procedure, his role is solely to adjudicate once the parties have presented their claims. Under the inquisitorial procedure, he plays an active role by first deciding whom he will hear.

It is standard in the literature to view accuracy in adjudication and procedural economy as the objectives at which legal procedures should aim; see for instance Sobel (1985), Froeb and Kobayashi (2001), and Parisi (2002). Adversarial systems of discovery clearly motivate parties to provide evidence. Nevertheless, they are often criticized (e.g., Tullock 1975, 1980) for yielding excessive expenditures through unnecessary duplication and costly overproduction of misleading information. We refer to legal procedures for concreteness. The same issues arise in regulatory or administrative hearings as well as in many other contexts.

One strand of literature has viewed the trial outcome as an exogenous 
function of the litigants' levels of effort or expenditure by using so-called success contest functions; see Cooter and Rubinfeld (1989), Farmer and Pecorino (1999), Katz (1988), and Parisi (2002). In these papers adjudication is a zeroone variable, i.e., a party either wins or loses. Parties engage in a rent-seeking game, leading to excessive expenditures. Our approach differs in that the arbiter's decisions are part of a perfect Bayesian equilibrium. In our set-up the arbiter is a sophisticated decision-maker who understands the parties' incentives to boost their claims.

In another well-known strand of literature, trials are modeled as persuasion games. Parties cannot falsify the verifiable evidence as such, but are able to misrepresent it by disclosing only what they see fit; see Sobel (1985), Milgrom and Roberts (1986), Lewis and Poitevin (1997), and Shin (1998). In our framework, by contrast, the parties do not have access to hard information; they dissipate resources in attempting to fabricate convincing stories.

Our paper is most closely related to the economics literature comparing adversarial with inquisitorial procedures of truth-finding. In this literature, "inquisitorial" usually refers to a system where a neutral investigator searches for evidence, "adversarial" to one where the parties to the conflict control the uncovering and presentation of evidence; see Shin (1998), Dewatripont and Tirole (1999), Froeb and Kobayashi (2001), and Palumbo (2001). However, in civil litigation and by contrast with criminal trials, the presentation of evidence essentially rests with the parties even in so-called inquisitorial systems. The main difference is the judge's involvement in controlling the litigants' presentation of evidence through bench requests, questions, and the like; see Langbein (1985) or Parisi (2002) for a comparative description, along these lines, of adversarial and inquisitorial systems. Demougin and Fluet (2008) present an analysis of active versus passive judging in a persuasion game set-up. They show that a more active or inquisitorial arbiter may eliminate inefficient equilibria. When presenting evidence involves a small cost, multiple equilibria typically exist under a purely adversarial procedure with a passive arbiter, some of which are more informative than others. Nev- 
ertheless, that paper does not deal with influence costs as such nor with the trade-off between submission costs and accuracy.

The paper is organized as follows. In the next section we describe our basic set-up. The following section derives the least-cost equilibrium. In the subsequent section we compare the adversarial procedure with the inquisitorial one. Section 5 concludes. Proofs are relegated to the Appendix.

\section{The Model}

A plaintiff $A$ has sued a defendant $B$ to obtain damages $x \in \mathbb{R}$. Damages are distributed with density $f(x)$ with full support over the real line. ${ }^{1}$ We assume $f(x)$ to be unimodal and symmetric; the mean is given by $\mu$ and the variance by $\sigma^{2}$. At the beginning of the trial all parties involved, i.e., plaintiff, defendant, and arbiter, know the distribution of $x$. The mean $\mu$ is such that, given the expected outcome of the trial, it is worthwhile for the defendant to sue. ${ }^{2}$

After the plaintiff has filed suit, both, plaintiff and defendant, learn the realization of $x$, meaning they become perfectly informed. The trial cannot be stopped at this point; the adjudicator has to decide the case. In particular, we rule out any out-of-court settlement negotiations.

The arbiter can adjudicate solely on the basis of his priors at that stage of the procedure as given by $f(x)$. Alternatively, he may receive further evidence submitted from the perfectly informed but self-interested plaintiff and defendant. The plaintiff $A$ would like the adjudicated value of $x$ to be large while the defendant $B$ would like it to be small. ${ }^{3}$

\footnotetext{
${ }^{1}$ We assume full support over the real line in order to avoid boundary conditions. The probability of extreme values of $x$ can be made, however, arbitrarily small.

${ }^{2}$ We will make this precise at the end of section 3.

${ }^{3}$ Throughout the text we take $x$ to be damages. Yet other examples abound: For example, in a divorce case $x$ may be the amount of support $A$ should get from $B$; in regulatory hearings $x$ may the rental charge for a local loop, the incumbent wants the charge to be high whereas the entrant wants it to be low.
} 
After plaintiff and defendant have learned $x$, they may testify in court. Testimony by the parties is costly. A submission is of the form "the value of the quantity at issue is $x_{i}$ ", $i=A, B$. It should be thought of as a story or argument rendering $x_{i}$ plausible, together with the supporting documents, witnesses, etc. The cost of a presentation is $\gamma+.5\left(x_{i}-x\right)^{2}, \quad i=A, B$, where $\gamma>0$. The actual value is $x$, which is observed by the party, and $x_{i}$ is the testimony or the statement submitted.

A distorting presentation is more costly than simply reporting the naked truth as it involves more fabrication. We take a quadratic function to capture the idea that the cost of misrepresenting the evidence increases at an increasing rate the further one moves away from the truth: it becomes more difficult to produce the corresponding documents or experts charge more the more they distort the truth. ${ }^{4}$

After the plaintiff and defendant have observed $x$, they decide simultaneously whether they do not testify, denoted by $\emptyset_{i}$, or whether they testify and report $x_{i}, i=A, B$. We denote their decision by $s_{i} \in S_{i}:=\left\{\emptyset_{i} \cup \mathbb{R}\right\}, i=A, B$. The cost is

$$
c_{i}\left(s_{i}, x\right)= \begin{cases}\gamma+.5\left(x_{i}-x\right)^{2}, & \text { if } s_{i}=x_{i} ; \\ 0, & \text { if } s_{i}=\emptyset_{i},\end{cases}
$$

$i=A, B$. Total submission cost is $C=c_{A}+c_{B}$.

The arbiter observes the defendant's and the plaintiff's choices and then adjudicates $\hat{x}\left(s_{A}, s_{B}\right)$. The arbiter is concerned about the loss from inaccuracy in adjudication and the parties' submission costs. Accordingly, there is a potential trade-off between procedural costs and the social benefits of correct adjudication. From the arbiter's perspective, the total social loss is

$$
L=l+C
$$

where $l$ is the loss from inaccurate adjudication or "error costs" and $C$ is

\footnotetext{
${ }^{4}$ Using quadratic falsification costs is standard in the literature. Maggi and RodríguezClare (1995) work with $c_{i}\left(x_{i}, x\right)=\gamma+\kappa\left(x_{i}-x\right)^{2}$ and interpret $\kappa$ as capturing the publicness of information. If $\kappa=0$, falsification is costless, therefore, information is purely private. As $\kappa$ increases, it becomes more costly to falsify information and for an arbitrarily large $\kappa$ the public-information model obtains.
} 
total submission cost.

Let $\hat{x}$ denote the arbiter's decision. The loss from inaccurate adjudication is

$$
l(\hat{x}, x)=\theta(\hat{x}-x)^{2}
$$

where $\theta>0$ is the rate at which the arbiter trades off accuracy against submission costs. If the true value is adjudicated, error costs are zero. The more the decision errs in either direction, the higher the losses from inaccurate adjudication and such losses increase at an increasing rate the further one moves away from the truth. The loss $l$ should be interpreted as the societal cost of incorrect decisions. For instance, in tort cases incorrect adjudication may have an adverse effect on deterrence.

The set-up is as follows. The parties observe $x$ and then simultaneously pick $s_{A}$ and $s_{B}$. The arbiter observes the parties' choices and then adjudicates $\hat{x}$.

The parties choose $s_{A}$ and $s_{B}$ so as to maximize $\pi_{A}$ and $\pi_{B}$ where

$$
\begin{aligned}
\pi_{A}\left(\hat{x}, s_{A}, x\right) & =\hat{x}-c_{A}\left(s_{A}, x\right) \text { and } \\
\pi_{B}\left(\hat{x}, s_{B}, x\right) & =-\hat{x}-c_{B}\left(s_{B}, x\right) .
\end{aligned}
$$

If the arbiter hears testimony, he updates his beliefs which denotes the probability distribution over $x$ in the information set given by the testimony. He then adjudicates $\hat{x}$ so as to minimize the loss from inaccurate adjudication. To satisfy the arbiter's concern about inaccurate decisions we look for equilibria where he infers the true $x$ if he hears testimony. To satisfy the arbiter's concern about submission costs, we pick out of the set of revealing equilibria the one where theses costs are minimal. We focus on symmetric perfect Bayesian equilibria.

\section{The least-cost equilibrium}

Suppose the plaintiff follows the strategy $s_{A}=\emptyset_{A}$ for $x<x_{A}^{0}$ and $s_{A}=x_{A}(x)$ otherwise; the defendant's strategy is $s_{B}=\emptyset_{B}$ for $x>x_{B}^{0}$ and $s_{B}=x_{B}(x)$ 
otherwise. Let $x_{i}$ be increasing and differentiable in $x$ if $i$ testifies alone or if they testify together, $i=A, B .^{5}$

We rule out totally unrevealing equilibria, meaning $x_{A}^{0}$ and/or $x_{B}^{0}$ are bounded. There is thus some range where at least one party sends a signal and $x$ is revealed. ${ }^{6}$

Next, we need some structure on out-of-equilibrium beliefs. We assume that at an out-of-equilibrium information set the arbiter believes that it was reached with the minimum number of deviations from the equilibrium strategies. A similar restriction on beliefs, termed the minimality condition has been used by Bagwell and Ramey (1991) or Emons and Fluet (2008, 2009).

First we show that the plaintiff and the defendant never testify together. together. The formal derivation of the following results are relegated to the Appendix.

Lemma 1: In equilibrium the plaintiff and the defendant never testify together, i.e., $x_{B}^{0}<x_{A}^{0}$.

To show this result we assume on the contrary that parties testify together. Yet it always pays for a party to deviate because this either changes the adjudicated value in his favor or signalling costs fall by more than revenues.

We can now state the least-cost signalling equilibrium. The equilibrium has the following structure:

\footnotetext{
${ }^{5}$ No party will ever signal over the entire support. If he signals, he incurs at least a cost of $\gamma$. He will not invest this amount to signal the arbiter that he is among the worst possible types.

${ }^{6}$ Under a properness restriction on the arbiter's out-of-equilibrium beliefs an equilibrium cannot be totally unrevealing (Myerson (1978)). To see this, suppose on the contrary that the arbiter believes $E(x)=\mu$ for all $s_{i} \in S_{i}, i=A, B$. If the parties actually choose not to testify, the arbiter's beliefs are borne out in equilibrium. Now suppose the arbiter observes some $x_{A} \gg \mu$. Sending signal $x_{A}$ is a "big" mistake for the type $\mu$ plaintiff and a "small" mistake for the type $x=x_{A}$ plaintiff at the proposed equilibrium. If the arbiter believes that big mistakes are less likely than small ones, he should conclude upon observing $x_{A}$ that the plaintiff is of type $x=x_{A}$.
} 
(i) If $x \in(\mu-\gamma, \mu+\gamma)$, neither party testifies; the arbiter believes and adjudicates $E\left(x \mid \emptyset_{A}, \emptyset_{B}\right)=\hat{x}\left(\emptyset_{A}, \emptyset_{B}\right)=\mu$, i.e., the prior mean.

(ii) If $x \geq \mu+\gamma$, the defendant plays $\emptyset_{B}$ and the plaintiff sends the signal $x_{A} \geq x$ solving

$$
x=x_{A}-1+e^{-\left(x_{A}-\mu-\gamma\right)} .
$$

The arbiter believes and adjudicates

$$
E\left(x \mid x_{A}, \emptyset_{B}\right)=\hat{x}\left(x_{A}, \emptyset_{B}\right)=x_{A}-1+e^{-\left(x_{A}-\mu-\gamma\right)}=x .
$$

(iii) If $x \leq \mu-\gamma$, the plaintiff plays $\emptyset_{A}$ and the defendant sends the signal $x_{B} \leq x$ solving

$$
x=x_{B}+1-e^{-\left(\mu-\gamma-x_{B}\right)} .
$$

The arbiter believes and adjudicates

$$
E\left(x \mid \emptyset_{A}, x_{B}\right)=\hat{x}\left(\emptyset_{A}, x_{B}\right)=x_{B}+1-e^{-\left(\mu-\gamma-x_{B}\right)}=x .
$$

To sum up:

Proposition 1: In the least-cost equilibrium if $x \leq \mu-\gamma$, the defendant sends the message $x_{B}$ solving (2) while the plaintiff is silent. If $x \in(\mu-\gamma, \mu+\gamma)$, neither party testifies. If $x \geq \mu+\gamma$, the plaintiff sends the message $x_{A}$ solving (1) and the defendant is silent. If one party testifies, the arbiter infers and adjudicates the true $x$; if both parties do not testify, the arbiter rationally expects and adjudicates $\mu$.

Let us now look at the message $x_{i}, i=A, B$ in more detail. Solving (1) and (2) yields

$$
\begin{aligned}
& x_{A}=x+1+\operatorname{ProductLog}\left(-e^{(\mu+\gamma-x-1)}\right) \text { and } \\
& x_{B}=x-1+\operatorname{ProductLog}\left(-e^{-(\mu-\gamma-x+1)}\right)
\end{aligned}
$$

where the ProductLog is the inverse function of $f(w)=w e^{w} \cdot{ }^{7}$ Consider, e.g., the plaintiff. We have $x_{A}(\mu+\gamma)=\mu+\gamma$, i.e., at the threshold $x_{A}^{0}=\mu+\gamma$ the

\footnotetext{
${ }^{7}$ See, e.g., http://en.wikipedia.org/wiki/Lambert's_W_function for more on the ProductLog.
} 
plaintiff signals the true $x$ at zero variable cost. For $x>x_{A}^{0}, x_{A}(x)>x$ and $\lim _{x \rightarrow \infty} x_{A}(x)=x+1$. The plaintiff boasts damages; boasting increases with damages, yet at a decreasing rate. See Figure 1. Except for the threshold, if the plaintiff testifies, he falsifies. For $x_{A}>x_{A}^{0}, E\left(x \mid x_{A}, \emptyset_{B}\right)$ is strictly monotone in $x_{A}$ : different $x$ 's give rise to different $x_{A}$ 's, to which the arbiter reacts by computing the correct expectation. Given $\partial E\left(x \mid x_{A}, \emptyset_{B}\right) / \partial x_{A} \neq 0$ and the marginal cost of lying is zero around the true value, it pays for the plaintiff to falsify if he testifies.

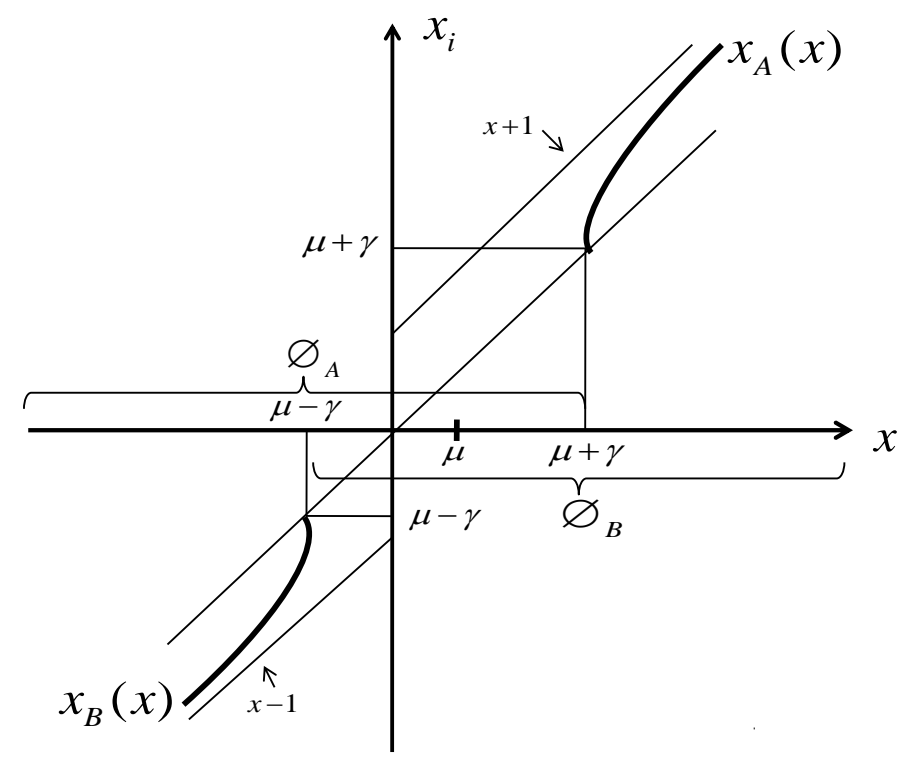

Figure 1: The least cost signalling strategies $x_{A}(x)$ and $\mathrm{x}_{B}(x)$

The social loss as seen by the adjudicator is

$$
\begin{aligned}
L^{A} & =\int_{-\infty}^{\mu-\gamma}\left(\gamma+.5\left(x-x_{B}\right)^{2}\right) f(x) d x+\theta \int_{\mu-\gamma}^{\mu+\gamma}(x-\mu)^{2} f(x) d x \\
& +\int_{\mu+\gamma}^{\infty}\left(\gamma+.5\left(x-x_{A}\right)^{2}\right) f(x) d x
\end{aligned}
$$

where the supersript $A$ indicates the adversarial procedure. By symmetry, the social loss can be rewritten as

$$
L^{A}=2 \theta \int_{\mu}^{\mu+\gamma}(x-\mu)^{2} f(x) d x+2 \int_{\mu+\gamma}^{\infty}\left(\gamma+.5\left(x-x_{A}\right)^{2}\right) f(x) d x .
$$


From a welfare point of view the adversarial procedure has the following virtues: when the value of information is small, the parties remain silent and do not spend resources on falsification, yet at the expense of incorrect decisions. Only when the value of information is sufficiently large, parties testify thus enabling correct decisions, yet at the expense of falsification. Note that the loss is a linear function of the error cost weight $\theta$ with the slope given by the error cost and the intercept given by the falsification cost.

Let us finally look at the plaintiff's decision to sue. The plaintiff's expected payoff in equilibrium is

$$
\begin{aligned}
E\left(\pi_{A}\right)= & \int_{-\infty}^{\mu-\gamma} x f(x) d x+\mu \int_{\mu-\gamma}^{\mu+\gamma} f(x) d x+ \\
& \int_{\mu+\gamma}^{\infty}\left(x-\gamma-.5\left(x-x_{A}\right)^{2}\right) f(x) d x>\mu-(\gamma+.5) \int_{\mu+\gamma}^{\infty} f(x) d x
\end{aligned}
$$

since $\left(x-x_{A}\right)<1$. The plaintiff sues if his expected payoff is positive. Thus, $\mu>(\gamma+.5) \int_{\mu+\gamma}^{\infty} f(x) d x$ is sufficient for the plaintiff to sue.

\section{Comparing the adversarial with the inquisi- torial procedure}

Let us now compare our least-cost equilibrium under the adversarial procedure with the least-cost equilibrium under the inquisitorial procedure which we derive in Emons and Fluet (2009). Under the inquisitorial procedure the

judge decides how much testimony he wants to hear. More specifically, in the first stage of the game we consider the arbiter announces whether he wants to hear no, one, or both parties. We denote this decision by $N, S, J$ where $N$ stands for no party being heard, $S$ for only a single party being heard (this would specify which one), and $J$ for joint submissions.

Under procedure $N$, no party testifies and submission costs are, therefore, zero. The arbiter then minimizes expected error costs solely on the basis of the priors implying $\hat{x}=\mu$. The expected total loss is $L_{N}^{I}=\theta \sigma^{2}$ where the superscript $I$ indicates the inquisitorial procedure. Obviously, $\theta \sigma^{2}$ is also 
the value of perfect information, given the accuracy $\sigma^{2}$ of the arbiter's prior information.

If only party $A$ is heard, $x_{A}(x)=x+1$ and $\hat{x}\left(x_{A}\right)=x_{A}-1$; if only party $B$ is heard $x_{B}(x)=x-1$ and $\hat{x}\left(x_{B}\right)=x_{B}+1$. The equilibrium is fully revealing and error costs are, therefore, zero. The expected loss $L_{S}^{I}=\gamma+.5$.

If both parties are required to testify $x_{A}(x)=x+.5, x_{B}(x)=x-.5$, and $\hat{x}\left(x_{A}, x_{B}\right)=.5 x_{A}+.5 x_{B}$. The equilibrium is fully revealing and error costs are again zero. The expected loss $L_{J}^{I}=2 \gamma+.25$.

Here the interesting result is that the extent of lying by, say, $B$ under single submission is twice the amount of his lying under joint submission. The reason is that under single submission greater weight is given to the party's report, thereby inducing him to falsify more. Thus, confronting the parties in hearings induces either of them to distort the evidence less than when only one testimony is heard. Given the quadratic cost of lying, this implies that the total variable cost of distorting is less under joint than under single submissions. Yet when both parties are heard, we have a duplication of the fixed submission cost $\gamma$.

Accordingly, under the inquisitorial procedure the arbiter has full control over which kind of costs he incurs: either only error costs (no testimony) or only falsification costs (single or joint testimony). The arbiter chooses whether no, only one party, or both parties are required to submit evidence so as to minimize the expected loss. For $\gamma \leq .25$, the optimal procedure is $J$ if $\theta \sigma^{2} \geq 2 \gamma+.25$ and $N$ otherwise; for $\gamma>.25$, the optimal procedure is $S$ if $\theta \sigma^{2} \geq \gamma+.5$ and $N$ otherwise. Figure 2 shows in the $(\theta, \gamma)$ plane the regions where the arbiter requires both, only one, or no party to submit evidence; in the figure we have set $\sigma^{2}=1$.

When the value of information is large (i.e., $\theta>.75$ ), the arbiter requires joint submissions if the fixed submission cost is sufficiently small, single submissions for intermediate values, and hears no one if the fixed cost is large. When $.25<\theta<.75$, the relevant choice is only between joint submissions or hearing no one: the value of information is then not large enough for single 
submissions to be worth it since they entail too much falsification. Finally, when $\theta<.25$, the value of information is too small even to compensate for the falsification costs under joint submissions.

Having described the outcome under the inquisitorial procedure, we now give sufficient conditions for one procedure to be better than the other one.

\section{Proposition 2:}

a) When $\theta$ is small or when $\theta$ is large, the expected loss under the inquisitorial procedure is lower than under the adversarial procedure.

b) Let $g(\theta):=(1+\sqrt{2 \theta+1}) / 2 \theta$. If $\gamma \geq g(\theta)$ and $\gamma \geq \theta-.5$ or if $\gamma \geq .25, \gamma \leq \theta-.5$, and $\gamma \leq g(\theta)$, the expected loss under the adversarial procedure is lower than under the inquisitorial procedure.

The proof for the superiority of the inquisitorial procedure is straightforward. When $\theta$ is small, no testimony is optimal under the inquisitorial procedure, leading to a social loss of $\theta \sigma^{2}$; see Figure 2. Obviously, the less the arbiter cares about accuracy, the smaller this loss becomes. The loss under the adversarial procedure as given by (3), is linear in $\theta$. Hence, even if $\theta=0$, society incurs the falsification costs with adversarial testimony.

If $\theta$ is sufficiently large, single or joint testimony is optimal under the inquisitorial procedure, leading to a loss of $\gamma+.5$ or $2 \gamma+.25$; see Figure 2. The equilibria are fully revealing and error costs are, therefore, zero. Under the adversarial procedure the parties do not testify over the interval $(\mu-\gamma, \mu+\gamma)$ leading to error costs that increase linearly with $\theta$. We may thus conclude: Under the inquisitorial procedure we have no falsification costs under no testimony and no error costs under single or joint testimony. Under adversarial testimony both falsification and error costs occur with positive probability. Thus, when the arbiter cares very little or very much about error costs, he does better with the inquisitorial procedure where he fully controls which kind of costs he incurs.

Let us now look at the cases where the adversarial procedure does better than the inquisitorial one. First we compare adversarial with no testimony. If 
$\gamma \geq g(\theta), L^{A}<L_{N}^{I}$. Over the interval $(\mu-\gamma, \mu+\gamma)$ our adversarial least-cost equilibrium and $N$ yield the same outcome - namely no testimony. In the tails $x \leq \mu-\gamma$ and $x \geq \mu+\gamma$, the adversarial procedure leads to the submission costs $\gamma+.5\left(x-x_{i}\right)^{2}<\gamma+.5$ since $\left|x-x_{i}\right|<1$; the inquisitorial procedure leads to the loss of inaccurate decisions $\theta(x-\mu)^{2}>\theta \gamma^{2}$. If $\gamma \geq g(\theta)$, submission costs are lower than the loss from inaccurate decisions. Submission costs increase linearly while error costs increase with the square of $\gamma$. Thus, for $\gamma$ sufficiently large, adversarial testimony is better than no testimony. In region $N$, no testimony is optimal under the inquisitorial procedure. Thus, when we are in region $N$ and $\gamma \geq g(\theta)$, the adversarial procedure is better than the inquisitorial one.

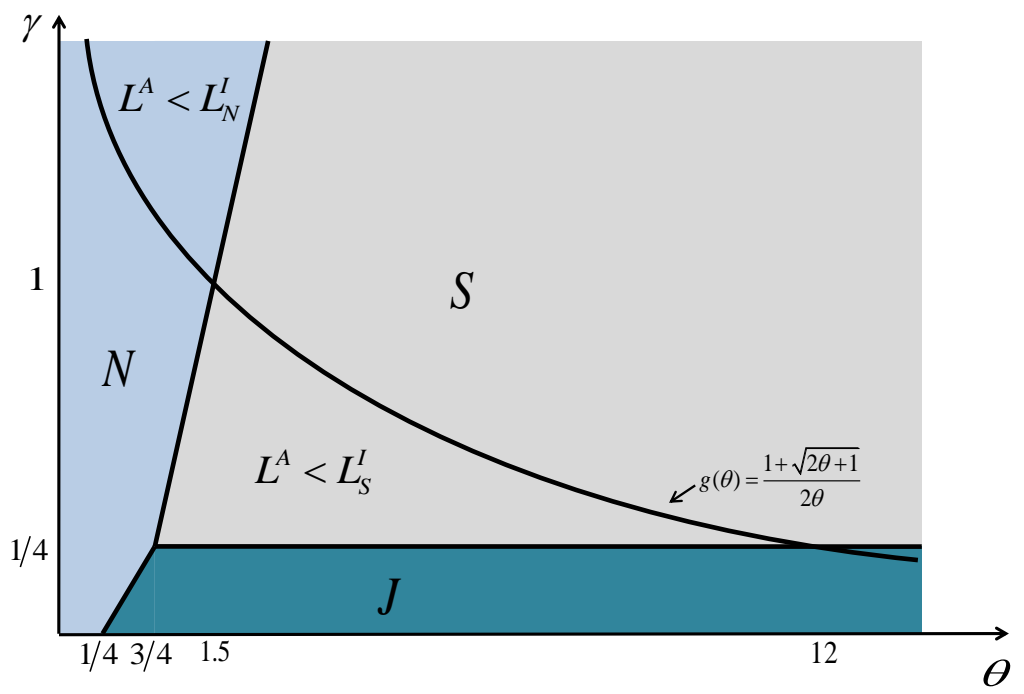

Figure 2: Optimal amount of testimony under the inquisitorial procedure

Next let us compare the adversarial outcome with single submission $S$. Here we have that if $\gamma \leq g(\theta), L^{A}<L_{S}^{I}$. Over the interval $(\mu-\gamma, \mu+\gamma)$ the adversarial procedure leads to the loss from inaccurate decisions $\theta(x-\mu)^{2}<$ $\theta \gamma^{2}$; in the tails the loss from boasting is $\gamma+.5\left(x-x_{i}\right)^{2}<\gamma+.5$. The loss from single testimony is $\gamma+.5$. For $\gamma$ sufficiently small, the loss from inaccurate 
decisions is smaller than the submission cost for $x \in(\mu-\gamma, \mu+\gamma)$. In region $S$, single testimony is optimal under the inquisitorial procedure. Thus, when we are in region $S$ and $\gamma \leq g(\theta)$, the adversarial procedure is better than the inquisitorial one.

Last consider joint submissions $J$. Under joint submissions for large values of $|x|$ the variable cost of falsifying of .25 is lower than the variable cost under the adversarial procedure. Yet, the fixed cost is duplicated. For $x \in[\mu-\gamma, \mu+\gamma]$ the error cost under the adversarial procedure have to be compared with the submission cost of $2 \gamma+.25$ of the inquisitorial procedure. A general comparison of joint submissions with the adversarial procedure turns out to be impossible.

We can, nevertheless, make the following argument. For $\theta \geq .75$ along the line $\gamma=.25$ single and joint testimony lead to the same expected loss which is, moreover, continuous in $\gamma$ for both procedures. From Proposition 2 we know that along this line for $\theta \in[.75,12]$ the adversarial procedure leads to losses strictly lower than single testimony. Therefore, by continuity, adversarial testimony also leads to lower losses than joint testimony for $\gamma=$ $.75-\epsilon, \theta \in[.75,12], \epsilon$ sufficiently small. ${ }^{8}$

Note that we have derived only sufficient conditions for one procedure to be better than the other one. Hence, for the areas other than the ones we have identified, we don't know which of the procedures is better.

To sum up: If the arbiter has strong views about submission costs, the inquisitorial procedure is better than the adversarial one, because the arbiter has full control about which kind of cost he incurs. If the arbiter is concerned about both, submission and falsification costs, the adversarial procedure may be better than the inquisitorial one because parties testify when the private and social value of information is high and do not testify when it is low.

\footnotetext{
${ }^{8}$ This type of argument can also be made for region $N(S)$ facing the area $S(N)$ where the adversarial procedure is better than the inquisitorial one.
} 


\section{Concluding Remarks}

We have derived the equilibrium testifying behavior under adversarial arbitration. When the true value of the amount at issue differs only slightly from the prior mean, the parties remain silent and do not spend resources on falsification. This comes at the expense of incorrect decisions, but the social loss from inaccurate adjudication will then also be small. Only when the true value differs sufficiently from the prior mean do parties testify. This enables correct decisions, yet at the expense of falsification costs.

Moreover, we have compared the adversarial with the inquisitorial procedure, taking into account submission costs and accuracy in adjudication. The inquisitorial procedure performs better when the arbiter has strong views about error costs; the adversarial procedure may perform better when the arbiter cares about both, error and falsification costs.

We have assumed extreme forms both for the adversarial and inquisitorial procedures. Under the former, our arbiter is passive and can just wait for testimony by the parties. Under the latter, the arbiter does not have the option to let the parties freely decide whether they want to testify: he can only either summon them to testify or refuse to hear them. Obviously, an active arbiter who also has the option to let the parties freely testify would yield the best of both worlds. On matters where accuracy has negligible social value, he would refuse to hear the parties. When accuracy has very large social value, he could summon one or both parties to testify. In intermediate cases, he could let the parties decide whether or not they want to testify. He then relies on the parties' superior private information about the true state to reach the best compromise between submission costs and accuracy. This is not unlike the justification often given for "managerial judges" who participate in activities such as pretrial discovery and settlement bargaining (see Schrag, 1999). 


\section{Appendix}

Proof of Lemma 1. Suppose on the contrary that there is some range $\left[x_{A}^{0}, x_{B}^{0}\right]$ where both parties testify and the equilibrium is revealing. At, say, $x_{B}^{0}$ the plaintiff $A$ reports $x_{A}\left(x_{B}^{0}\right)$, the defendant $B$ signals $x_{B}\left(x_{B}^{0}\right)$, and $E\left(x \mid x_{A}\left(x_{B}^{0}\right), x_{B}\left(x_{B}^{0}\right)\right)=x_{B}^{0}$. See Figure 6.

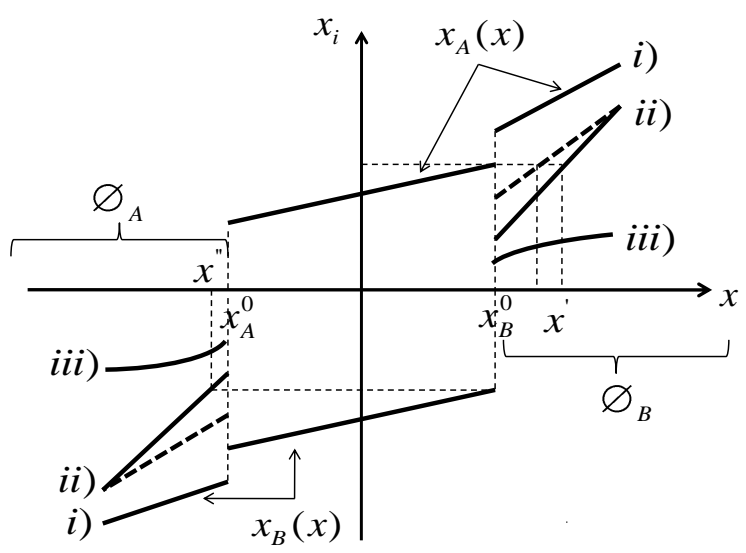

Figure 3: Signalling strategies $x_{A}(x)$ and $\mathrm{x}_{B}(x)$ if $x_{A}^{0}<x_{B}^{0}$

We have to distinguish three cases.

i) Let $x_{A}\left(x_{B}^{0}\right) \leq \lim _{\epsilon \rightarrow 0} x_{A}\left(x_{B}^{0}+\epsilon\right)$. Here there is no signal $x_{i}$ which is sent by party $i$ for different two different values of $x$. The argument depends on whether the plaintiff's signalling costs go up or down when he switches from $x_{A}\left(x_{B}^{0}\right)$ to $x_{A}\left(x_{B}^{0}+\epsilon\right)$ given $x=x_{B}^{0}$. For $\epsilon$ sufficiently small, the sign of the cost change is the same if $x=x_{B}^{0}+\epsilon$.

Consider first the case where the plaintiff's cost does not increase when he switches from $x_{A}\left(x_{B}^{0}+\epsilon\right)$ to $x_{A}\left(x_{B}^{0}\right)$. Let the true state be $x_{B}^{0}+\epsilon$. Along the equilibrium path $\left(x_{A}\left(x_{B}^{0}+\epsilon\right), \emptyset_{B}\right)$ and the arbiter correctly infers $x_{B}^{0}+\epsilon$.

If the plaintiff deviates to $x_{A}\left(x_{B}^{0}\right)$, the arbiter observes $\left(x_{A}\left(x_{B}^{0}\right), \emptyset_{B}\right)$ which is off the equilibrium. By the minimality condition the judge thinks that either the defendant deviated while the plaintiff played his equilibrium action and the underlying $x=x_{B}^{0}$; or he thinks that the defendant played his equilibrium action $\emptyset_{B}$ while the plaintiff deviated and the underlying $x=\int_{x_{B}^{0}}^{\infty} x f(x) d x / \int_{x_{B}^{0}}^{\infty} f(x) d x>$ $x_{B}^{0}+\epsilon$ for $\epsilon$ small. The arbiter assigns equal probability to both possibilities so that $E\left(x \mid x_{A}\left(x_{B}^{0}\right), \emptyset_{B}\right)>x_{B}^{0}+\epsilon$. Since the plaintiff's cost does not increase and his revenue increases, he will deviate. 
Now consider the case where the plaintiff's costs decrease when he switches from $x_{A}\left(x_{B}^{0}\right)$ to $x_{A}\left(x_{B}^{0}+\epsilon\right)$. Let the true state be $x_{B}^{0}$. Along the equilibrium path $\left(x_{A}\left(x_{B}^{0}\right), x_{B}\left(x_{B}^{0}\right)\right)$ and the arbiter correctly infers $x_{B}^{0}$.

Suppose the plaintiff switches to $x_{A}\left(x_{B}^{0}+\epsilon\right)$ so that the arbiter observes $\left(x_{A}\left(x_{B}^{0}+\epsilon\right), x_{B}\left(x_{B}^{0}\right)\right)$ which is off the equilibrium. Then the judge thinks that the plaintiff deviated and $x=x_{B}^{0}$ or the defendant deviated and $x=x_{B}^{0}+\epsilon$. Thus, $E\left(x \mid x_{A}\left(x_{B}^{0}+\epsilon, x_{B}\left(x_{B}^{0}\right)\right)=x_{B}^{0}+.5 \epsilon\right.$. The plaintiff's costs decrease, his revenue does not decrease, hence he will deviate.

ii) Let $x_{A}\left(x_{B}^{0}\right)>\lim _{\epsilon \rightarrow 0} x_{A}\left(x_{B}^{0}+\epsilon\right)$ and $\lim _{x \rightarrow \infty} x_{A}(x) \geq x_{A}\left(x_{B}^{0}\right)$. Here there are signals $x_{i}$ which are sent by party $i$ for two different values of $x$.

Consider first the case where $x_{A}\left(x_{A}^{0}\right)<x_{A}\left(x_{B}^{0}+\epsilon\right)$ or, by symmetry, $x_{B}\left(x_{B}^{0}\right)>$ $x_{B}\left(x_{A}^{0}-\epsilon\right)$ which is given by the dotted line in Figure 6. Here $x_{B}\left(x_{B}^{0}\right)$ is played by $B$ only at $x_{B}^{0}$ in equilibrium. Let $x^{\prime}>x_{B}^{0}$ be such that $x_{A}\left(x^{\prime}\right)=x_{A}\left(x_{B}^{0}\right)$. Now suppose $A$ lowers the signal to $x_{A}\left(x_{B}^{0}\right)-\epsilon$ so that the judge observes $\left(x_{A}\left(x_{B}^{0}\right)-\right.$ $\left.\epsilon, x_{B}\left(x_{B}^{0}\right)\right)$ which is off the equilibrium path. Then he thinks with equal probability that either $A$ deviated and $x=x_{B}^{0}$ or $B$ deviated and $x=x_{B}^{0}-\epsilon$ or $x=x^{\prime}-\delta$; let the arbiter assign probabilities $b>0$ and $(1-b)$ to the two possibilities. Hence, $E\left(x \mid x_{A}\left(x_{B}^{0}\right)-\epsilon, x_{B}\left(x_{B}^{0}\right)\right)=.5 x_{B}^{0}+.5\left(b\left(x_{B}^{0}-\epsilon\right)+(1-b)\left(x^{\prime}-\delta\right)\right)$. For $\epsilon$ going to zero, so does $\delta$ and $\lim _{\epsilon \rightarrow 0} E\left(x \mid x_{A}\left(x_{B}^{0}\right)-\epsilon, x_{B}\left(x_{B}^{0}\right)\right)>x_{B}^{0}$. A's revenue increases, hence he will deviate.

Now consider the case where $x_{A}\left(x_{A}^{0}\right)>x_{A}\left(x_{B}^{0}+\epsilon\right)$ or, by symmetry, $x_{B}\left(x_{B}^{0}\right)<$ $x_{B}\left(x_{A}^{0}-\epsilon\right)$. Here any equilibrium signal $x_{i}$ under joint testifying is also played when $i$ testifies alone. Let $x^{\prime \prime}<x_{A}^{0}$ be such that $x_{B}\left(x^{\prime \prime}\right)=x_{B}\left(x_{B}^{0}\right)$.

Suppose the true state is $x_{B}^{0}$. Along the equilibrium path $\left(x_{A}\left(x_{B}^{0}\right), x_{B}\left(x_{B}^{0}\right)\right)$ and the judge infers $x_{B}^{0}$. If $A$ lowers his signal to $x_{A}\left(x_{B}^{0}\right)-\epsilon$, the judge observes $\left(x_{A}\left(x_{B}^{0}\right)-\epsilon, x_{B}\left(x_{B}^{0}\right)\right)$ which is off the equilibrium. Then the arbiter thinks with probability .5 that $A$ deviated and $x=x_{B}^{0}$ or $x=x^{\prime \prime}$; the judge assigns probabilities $b$ and $(1-b)$ to theses possibilities. With probability .5 he thinks that $B$ deviated and $x=x_{B}^{0}-\epsilon$ or $x=x^{\prime}-\delta$. Thus, $E\left(x \mid x_{A}\left(x_{B}^{0}\right)-\epsilon, x_{B}\left(x_{B}^{0}\right)\right)=.5\left(b x_{B}^{0}+(1-\right.$ b) $\left.x^{\prime \prime}\right)+.5\left(b\left(x_{B}^{0}-\epsilon\right)+(1-b)\left(x^{\prime}-\delta\right)\right.$. We have $\lim _{\epsilon \rightarrow 0} E\left(x \mid x_{A}\left(x_{B}^{0}\right)-\epsilon, x_{B}\left(x_{B}^{0}\right)\right)=$ $b x_{B}^{0}+.5(1-b)\left(x^{\prime}+x^{\prime \prime}\right)$. If this exceeds $x_{B}^{0}, A$ deviates.

If $B$ lowers his signal to $x_{B}\left(x_{B}^{0}\right)-\epsilon$, the judge observes $\left(x_{A}\left(x_{B}^{0}\right), x_{B}\left(x_{B}^{0}\right)-\right.$ $\epsilon)$ which is off the equilibrium. Then he thinks that $B$ deviated and $x=x_{B}^{0}$ or $x=x^{\prime}$; or he thinks $A$ deviated and $x=x_{B}^{0}-\epsilon$ or $x=x^{\prime \prime}-\delta$. Hence, $E\left(x \mid x_{A}\left(x_{B}^{0}\right), x_{B}\left(x_{B}^{0}\right)-\epsilon\right)=.5\left(b x_{B}^{0}+(1-b) x^{\prime}\right)+.5\left(b\left(x_{B}^{0}-\epsilon\right)+(1-b)\left(x^{\prime \prime}-\delta\right)\right)$. Again we have $\lim _{\epsilon \rightarrow 0} E\left(x \mid x_{A}\left(x_{B}^{0}\right), x_{B}\left(x_{B}^{0}\right)-\epsilon\right)=b x_{B}^{0}+.5(1-b)\left(x^{\prime}+x^{\prime \prime}\right)$. If this is less than $x_{B}^{0}, B$ will deviate.

iii) Let $x_{A}\left(x_{B}^{0}\right)>\lim _{\epsilon \rightarrow 0} x_{A}\left(x_{B}^{0}+\epsilon\right)$ and $\lim _{x \rightarrow \infty} x_{A}(x)<x_{A}\left(x_{B}^{0}\right)$. Here again there is no signal $x_{i}$ which is sent by party $i$ for two different values of $x$ and the argument is along the same lines as in i). 
Proof of Proposition 1. The equilibrium strategies satisfy the following conditions:

(i) There exists $x_{A}^{0}>x_{B}^{0}$ such that $A$ plays $s_{A}=\emptyset_{A}$ if $x<x_{A}^{0}$ and $s_{A}=x_{A}$ otherwise, for some $x_{A}$ yet to be determined; $B$ plays $s_{B}=\emptyset_{B}$ if $x>x_{B}^{0}$ and $s_{B}=x_{B}$ otherwise, for some $x_{B}$ yet to be determined.

(ii) The adjudicator's beliefs are denoted $\varphi\left(s_{A}, s_{B}\right)$. Along the equilibrium path these beliefs are the updated expectation of $x$ given the parties' actions, i.e., $\varphi\left(s_{A}, s_{B}\right)=E\left(x \mid s_{A}, s_{B}\right)$. The adjudicator's seeks to minimize the expected quadratic error. Hence, his sequentially rational strategy is $\hat{x}=\varphi\left(s_{A}, s_{B}\right)$.

Priors are represented by the density $f(x)$ which is unimodal and symmetric with mean $\mu$ and variance by $\sigma^{2}$. We focus on equilibria where when one party submits and the true $x$ is inferred by the adjudicator.

Consider first the case $x \geq x_{A}^{0}$. Then only $A$ signals and the equilibrium strategies satisfy

$$
\begin{aligned}
& \partial \pi_{A}\left(\varphi\left(x_{A}, \emptyset_{B}\right), x_{A}, x\right) / \partial x_{A}=\partial \varphi\left(x_{A}, \emptyset_{B}\right) / \partial x_{A}-\left(x_{A}-x\right)=0 \\
& \text { and } \varphi\left(x_{A}, \emptyset_{B}\right)=x \quad \text { for all } x \geq x_{A}^{0} .
\end{aligned}
$$

Combining both conditions yields the differential equation

$$
\partial \varphi\left(x_{A}, \emptyset_{B}\right) / \partial x_{A}-\left(x_{A}-\varphi\left(x_{A}, \emptyset_{B}\right)\right)=0 \quad \text { for all } x \geq x_{A}^{0} .
$$

The strategy $x_{A}$ is then a global maximum for party $A$; see Mailath (1987). The general solution for the differential equation (4) is

$$
\varphi\left(x_{A}, \emptyset_{B}\right)=K e^{-x_{A}}+x_{A}-1 .
$$

Signalling costs (excluding the fixed cost) are

$$
.5\left(x_{A}-\varphi\left(x_{A}, \emptyset_{B}\right)\right)^{2}=.5\left(1-K e^{-x_{A}}\right)^{2} .
$$

The "smallest" message sent by $A$ occurs when $x=x_{A}^{0}$. Signalling costs in that state of the world are minimized if $x_{A}\left(x_{A}^{0}\right)=x_{A}^{0}$. This requires $K=e^{x_{A}^{0}}$.

Substituting in (5) and recalling that $\left.\varphi\left(x_{A}(x), \emptyset_{B}\right)\right) \equiv x$ then yields

$$
x=x_{A}-1+e^{-\left(x_{A}-x_{A}^{0}\right)} \quad \text { for all } x \geq x_{A}^{0} .
$$

The solution $x_{A}$ to this equation is party $A$ 's signalling strategy. The constant $x_{A}^{0}$ remains to be determined.

Consider next the case where $x \leq x_{B}^{0}$. Now only $B$ submits. The argument is similar to the one above, except that $B$ wants to persuade the adjudicator that $x$ is small. We obtain:

$$
x=x_{B}+1-e^{-\left(x_{B}^{0}-x_{B}\right)} \quad \text { for all } x \leq x_{B}^{0} .
$$


The solution $x_{B}$ to this equation is party $B$ 's signalling strategy.

It remains to determine the constants $x_{A}^{0}$ and $x_{B}^{0}$. When neither party submits, the arbiter's beliefs are

$$
\varphi_{\emptyset}:=E\left(x \mid \emptyset_{A}, \emptyset_{B}\right)=E\left(x \mid x_{B}^{0}<x<x_{A}^{0}\right) .
$$

At $x=x_{A}^{0}$ party $A$ is just indifferent between submitting and not submitting. If the party submits, the true state is revealed at the cost of $\gamma$. If the party does not submit, the arbiter adjudicates $\varphi_{\emptyset}$. Thus party $A$ is indifferent if $x_{A}^{0}-\gamma=\varphi_{\emptyset}$. Using the same argument, when $x=x_{B}^{0}$, party $B$ is indifferent between submitting and not if $-x_{B}^{0}-\gamma=-\varphi_{\emptyset}$. Combining with (8) yields

$$
\varphi_{\emptyset}=E\left(x \mid \varphi_{\emptyset}-\gamma<x<\varphi_{\emptyset}+\gamma\right) .
$$

Thus, the updated expectation given that $x$ is in the interval $\left[\varphi_{\emptyset}-\gamma, \varphi_{\emptyset}+\gamma\right]$ must be the mid point $\varphi_{\emptyset}$. With a symmetric unimodal prior density this is possible only if $\varphi_{\emptyset}$ equals the prior mean $\mu$.

Consequently, $x_{A}^{0}=\mu+\gamma, x_{B}^{0}=\mu-\gamma$. The equilibrium signalling strategies, therefore, satisfy

$$
\begin{array}{ll}
x=x_{A}-1+e^{-\left(x_{A}-\mu-\gamma\right)} & \text { for all } x \geq \mu+\gamma \quad \text { and } \\
x=x_{B}+1-e^{-\left(\mu-\gamma-x_{B}\right)} & \text { for all } x \leq \mu-\gamma
\end{array}
$$

Proof of Proposition 2 b).

For the loss under the adversarial procedure as given by (3) we have

$$
L^{A}<2 \theta \int_{\mu}^{\mu+\gamma}(x-\mu)^{2} f(x) d x+2(\gamma+.5) \int_{\mu+\gamma}^{\infty} f(x) d x
$$

since $x-x_{A}<1$.

For the loss under no testimony we have

$$
L_{N}^{I} \geq 2 \theta \int_{\mu}^{\mu+\gamma}(x-\mu)^{2} f(x) d x+2 \theta \gamma^{2} \int_{\mu+\gamma}^{\infty} f(x) d x
$$

because $(x-\mu)^{2}>\gamma^{2}$ for $x \geq \mu+\gamma$. Therefore, if $\gamma>g(\theta), L^{A}<L_{N}^{I}$.

Using (9), since $(x-\mu)^{2} \leq \gamma^{2}$ for $\in[\mu, \mu+\gamma]$, we have

$$
L^{A}<2 \theta \gamma^{2} \int_{\mu}^{\mu+\gamma} f(x) d x+2(\gamma+.5) \int_{\mu+\gamma}^{\infty} f(x) d x .
$$

Hence, if $\gamma<g(\theta), L^{A}<L_{S}^{I}=\gamma+.5$. 


\section{References}

Bagwell, Kyle and Garey Ramey. 1991. "Oligopoly Limit Pricing," 22 Rand Journal of Economics 155-72.

Bernardo, Antonio E., Eric Talley, and Ivo Welch. 2000. "A Theory of Legal Presumptions," 16 Journal of Law, Economics, and Organization 1-49.

Cooter, Robert and Daniel L. Rubinfeld. 1989. "Economic Analysis of Legal Disputes and their Resolution," 27 Journal of Economic Literature 1067-1097.

Demougin, Dominique and Claude Fluet. 2008. "Rules of Proof, Courts, and Incentives," 39 Rand Journal of Economics 20-40.

Dewatripont, Mathias and Jean Tirole. 1999. "Advocates," 107 Journal of Political Economy 1-39.

Emons, Winand and Claude Fluet. 2008. "Non-comparative versus Comparative Advertising as a Quality Signal," University of Bern, Dept. of Economics Discussion Paper 08.05, www.staff.unibe.ch/emons/downloads/ad_vers3.pdf.

Emons, Winand and Claude Fluet. 2009. "Accuracy versus Falsification Costs: The Optimal Amount of Evidence under different Procedures," 25 Journal of Law, Economics, and Organization 134-156.

Farmer, Amy and Paul Pecorino. 1999. "Legal Expenditure as a Rent-Seeking Game," 100 Public Choice 271-288.

Froeb, Luke and Bruce Kobayashi. 2001. "Evidence Production in Adversarial vs. Inquisitorial Regimes," 72 Economics Letters 267-272.

Katz, Avery. 1988. "Judicial Decisionmaking and Litigation Expenditure," 8 International Review of Law and Economics 127-143.

Langbein, John. 1985. "The German Advantage in Civil Procedure," 52 University of Chicago Law Review 823-866.

Lewis, Tracy and Michael Poitevin. 1997. "Disclosure of Information in Regulatory Proceedings," 13 Journal of Law, Economics, and Organization 50-73.

Maggi, Giovanni and Andrés Rodríguez-Clare. 1995 "Costly Distortion of Information in Agency Problems," 26 Rand Journal of Economics 675-689.

Mailath, George. 1987 "Incentive Compatibility in Signaling Games with a Continuum of Types," 55 Econometrica 1349-1365.

Milgrom, Paul and John Roberts. 1986. "Relying on the Information of Interested Parties," 17 Rand Journal of Economics 18-32.

Myerson, Roger. 1978. "Refinements of the Nash Equilibrium Concept," 7 International Journal of Game Theory 73-80.

Palumbo, Giuliana. 2001. "Trial Procedures and Optimal Limits on ProofTaking," 21 International Review of Law and Economics 309-327.

Parisi, Francesco. 2002. "Rent-seeking through Litigation: Adversarial and Inquisitorial Systems Compared," 22 International Review of Law and Economics 
193-216.

Schrag, Joel L. 1999. "Managerial Judges: An Economic Analysis of Judicial Management of Legal Discovery," 30 Rand Journal of Economics 305-323.

Shin, Hyun S. 1998. "Adversarial and Inquisitorial Procedures in Arbitration," 29 Rand Journal of Economics 378-405.

Sobel, Joel. 1985. "Disclosure of Evidence and Resolution of Disputes: Who Should Bear the Burden of Proof?," in A. E. Roth, ed., Game Theoretic Models of Bargaining, Cambridge: Cambridge University Press, 341-361.

Tullock, Gordon. 1975. "On the Efficient Organization of Trials," 28 Kyklos 745762 .

Tullock, Gordon. 1980, Trials on Trials: The Pure Theory of Legal Procedure. New York: Columbia University Press. 